\title{
Campylobacter jejuni isolated from poultry and humans in Styria, Austria: epidemiology and ciprofloxacin resistance
}

\author{
I. HEIN ${ }^{1 *}$, C. SCHNECK ${ }^{1}$, M. KNÖGLER ${ }^{1}$, G. FEIERL ${ }^{2}$, P. PLESS $^{3}$, J. KÖFER $^{3}$, \\ R. ACHMANN ${ }^{4}$ AND M. WAGNER ${ }^{1}$ \\ ${ }^{1}$ Institute of Milk Hygiene, Milk Technology, and Food Science, University of Veterinary Medicine, \\ Veterinaerplatz 1, A-1210 Vienna, Austria \\ ${ }^{2}$ Institute of Hygiene, Karl-Franzens University Graz, Universitaetsplatz 1, A-8010 Graz, Austria \\ ${ }^{3}$ Department of Veterinary Administration in Styria, Zimmerplatzgasse 15, A-8010 Graz, Austria \\ ${ }^{4}$ Institute of Animal Breeding and Genetics, University of Veterinary Medicine, Veterinaerplatz 1, \\ A-1210 Vienna, Austria
}

(Accepted 9 January 2003)

\section{SUMMARY}

Sixty-six broiler flocks were sampled to determine the presence of Campylobacter spp. at slaughter in 1998. Thirty flocks (45\%) tested positive and C. jejuni was identified in all isolates. Combined pulsed-field gel electrophoresis/amplified fragment length polymorphism (PFGE/ AFLP) subtyping of 177 isolates from 24 positive flocks revealed 62 subtypes; 16 flocks harboured more than one subtype. When subtyping 101 clinical $C$. jejuni isolates collected in the same time period and area, 60 PFGE/AFLP types were identified. Comparison of subtypes from poultry and human isolates revealed three shared PFGE/AFLP types, which were present in 11 human isolates. Fifty per cent of all poultry isolates and $39 \%$ of all human isolates were resistant to ciprofloxacin. The present study confirms the increase in ciprofloxacin resistance in both human and poultry C. jejuni isolates in Austria, as observed in several countries worldwide. A small number of human isolates shared PFGE/AFLP types with poultry isolates, however, further studies should also focus on the identification of other sources of $C$. jejuni infection in humans.

\section{INTRODUCTION}

Thermophilic Campylobacter spp., mainly Campylobacter jejuni, have been recognized as a major cause of human gastroenteritis throughout the world. In Austria, the incidence of campylobacter infections, based on laboratory-confirmed cases, was 70 per 100000 inhabitants, as determined in a national survey in the federal province of Styria (1.2 million inhabitants) in 2000 [1]. In Austria, following Salmonella spp., C. jejuni is the second most common foodborne bacterial pathogen that is known to cause

\footnotetext{
* Author for correspondence.
}

diarrhoea. Contaminated raw milk, drinking water and poultry are considered to be the main sources of infection, both in outbreaks and in sporadic cases [2]. Campylobacter spp. have been isolated from up to $82 \%$ of broiler flocks at slaughter [3-6].

Quinolones are frequently used for the treatment of campylobacteriosis in humans but the prevalence of resistance of Campylobacter spp. to these antibiotics has increased in several countries. For instance, in Austria the resistance of clinical Campylobacter spp. isolates to ciprofloxacin increased from $0 \%$ in 1988 to $34 \cdot 1 \%$ in 1997 [7-9]. Several researchers attribute this pattern to the introduction of quinolones in poultry production $[10,11]$. 
For epidemiological purposes, several molecular typing methods of Campylobacter spp., such as the random amplified polymorphic DNA (RAPD) technique, the restriction fragment length polymorphism (RFLP) technique, and pulsed-field gel electrophoresis (PFGE) have been used [12-18]. PFGE was shown to be a highly discriminatory and reproducible technique, especially when two or more different restriction enzymes were applied [19]. The amplified fragment length polymorphism (AFLP) technique is also a highly discriminatory method of subtyping Campylobacter spp. [20-22].

The aims of the present study were $(a)$ to assess the prevalence of Campylobacter spp. resistant and sensitive to ciprofloxacin in poultry flocks at slaughter in the Austrian district of Styria over a period of 3 months; (b) to investigate the percentage of ciprofloxacin-resistant and ciprofloxacin-sensitive clinical Campylobacter spp. isolates collected in the same area and time period; and (c) to compare the PFGE and AFLP types of poultry and clinical isolates in order to estimate their genetic similarity.

\section{METHODS}

\section{Sample collection}

Sixty-six broiler flocks of the eastern and southern parts of Styria, Austria, which were slaughtered from October 1998 to December 1998 were sampled at slaughter by the Department of Veterinary Administration in Graz (Styria), Austria, to determine the presence of Campylobacter spp. The average flock size was 20000 chickens per flock. In regular time intervals during the slaughtering process the intestines of a total of 10 chickens per flock were collected from the eviscerating line. The samples were placed into sterile plastic bags, stored at $4{ }^{\circ} \mathrm{C}$, transported to the laboratory in chilled boxes, and analysed within $24 \mathrm{~h}$. Caecal contents were removed aseptically, cultured on modified Preston Agar (mCCDA, Oxoid, Basingstoke, UK) supplemented with CCDA Selective Supplement Code SR 155E (Oxoid), and incubated under microaerobic conditions (GENbox Microaer, BioMérieux, Marcy l'Etoile, France) at $42^{\circ} \mathrm{C}$ for $48 \mathrm{~h}$. Cultures suspected to contain Campylobacter spp. were sent on mCCDA to the Institute of Hygiene, University of Graz, Austria, for further confirmation. Generally, one colony per plate was picked for further confirmation. In parallel, 239 C. jejuni strains were included in the study; the strains were isolated from human faecal samples collected in the same time period and area by local practitioners and in hospitals.

\section{Genus and species identification}

Strains were subcultured on blood agar (BioMérieux) under microaerobic conditions at $42{ }^{\circ} \mathrm{C}$ for $48 \mathrm{~h}$. Genus and species identity was confirmed by inspection of colony morphology on mCCDA, Gram stain, detection of catalase activity, cytochrome oxidase production and hydrolysis of hippurate. Strains that did not hydrolyse hippurate were subjected to PCR targeting the flaA gene [23] and the hip gene [24].

\section{Antimicrobial susceptibility testing}

Susceptibility testing to ciprofloxacin ( $5 \mu \mathrm{g}$ disk) was performed by using the agar disk diffusion method [25] on Mueller-Hinton agar supplemented with $5 \%$ defibrinated sheep blood (BioMérieux). The plates were inoculated with bacterial cultures adjusted to a $0.5 \mathrm{McFarland}$ standard under microaerobic conditions at $42{ }^{\circ} \mathrm{C}$ for $24-48 \mathrm{~h}$. Isolates showing an inhibition zone diameter $\leqslant 15 \mathrm{~mm}$ were designated as resistant, and $\geqslant 21 \mathrm{~mm}$ as sensitive. Ciprofloxacin sensitive C. jejuni ATCC 33560 and C. coli ATCC 33559 as well as a ciprofloxacin resistant $C$. jejuni CIP 105890 were used as control strains.

\section{Subtyping of $C$. jejuni isolates by PFGE}

Subtyping of $C$. jejuni isolates by PFGE was performed as published previously [16], using the restriction enzymes SmaI and SalI (New England Biolabs, Beverly, MA, USA). Macrorestriction profiles were visualized after gels were stained with ethidium bromide under UV light and stored as digitized images in TIFF format.

To facilitate the visual analysis of the profiles, computerized distance estimation and cluster analysis was performed. Normalization according to molecular weight standards on the outermost lanes of each gel and conversion of band patterns to binary data were done using RFLPscan ${ }^{\mathrm{TM}}$ software (Scanalytics, Billerica, MA, USA). For estimation of distance according to the coefficient of Link et al. [26] and cluster analysis using the unweighted-pair group method (UPGMA), the TREECON software package (version 1.2, Yves van de Peer, Royal University of Antwerp, Belgium) was applied. Two isolates shared one PFGE type if the combined SmaI/SalI patterns were completely identical. 
Table 1. Sequences and modifications of adapter and primer oligonucleotides used for AFLP

\begin{tabular}{ll}
\hline \hline Oligonucleotide & Sequence and modification \\
\hline HindIII-specific adapter & 5'-CTC GTA GAC TGC GTA CC-NH ${ }_{2}-3^{\prime}$ \\
& 5'-P-AGC TGG TAC GCA GTC-3' \\
Hhal-specific adapter & 5'-GAC GAT GAG TCC TGA TCG-3' \\
& 5'-ATC AGG ACT CAT CG-NH ${ }_{2}-3^{\prime}$ \\
Hin $\mathrm{dIII}+0$ & 5'-GAC TGC GTA CCA GCT T-3' \\
HindIII + A & 5'-FAM-GAC TGC GTA CCA GCT TA-3' \\
HhaI +0 & 5'-GAT GAG TCC TGA TCG C-3' \\
HhaI + A & 5'-GAT GAG TCC TGA TCG CA-3' \\
\hline \hline
\end{tabular}

\section{Subtyping of $C$. jejuni isolates by AFLP}

\section{Restriction and ligation}

AFLP was performed as published previously [20]. Genomic DNA was extracted from undigested PFGE agarose plugs using the QIAquick ${ }^{\mathrm{TM}}$ Gel Extraction Kit (Qiagen Inc., Valencia, CA, USA) according to the manufacturer's instructions. A total of $25 \mathrm{ng}$ of DNA were digested and ligated in a volume of $14 \mu 1$ containing $5 \mathrm{U}$ of HindIII, $5 \mathrm{U}$ of $H h a \mathrm{I}$, and $1 \mathrm{U}$ T4 DNA ligase (New England Biolabs), $570 \mathrm{~nm}$ HindIII- and $5.7 \mu \mathrm{M}$ HhaI-restriction site-specific adapter, $36 \mathrm{~mm} \mathrm{NaCl}, 54 \mathrm{~mm}$ Tris- $\mathrm{HCl}$ (pH 7.8), $11 \mathrm{~mm} \mathrm{MgCl}_{2}, 11 \mathrm{~mm}$ DTT, $1 \mathrm{~mm}$ ATP, and $63 \mathrm{ng} / \mu \mathrm{l}$ BSA for $2 \mathrm{~h}$ at $37^{\circ} \mathrm{C}$. The adapters were modified to allow exponential amplification of heterosite fragments only (Table 1) [27]. A total of $186 \mu 1$ distilled $\mathrm{H}_{2} \mathrm{O}$ were added to the restriction ligation mixture.

\section{PCR}

Primers used for preselective and selective PCR are listed in Table 1. Preselective PCR was performed in a $20 \mu \mathrm{l}$ volume containing $10 \mathrm{~mm}$ Tris $-\mathrm{HCl}, 50 \mathrm{~mm}$ $\mathrm{KCl}, 2.5 \mathrm{mM} \mathrm{MgCl}_{2}, 0.001 \%$ (w/v) gelatin, $250 \mathrm{~nm}$ adapter-specific primer HindIII $+0,2.5 \mu \mathrm{M}$ adapterspecific primer $H h a \mathrm{I}+0,200 \mu \mathrm{M}$ each of dATP, dTTP, dGTP and dCTP, 1.25 U AmpliTaq Gold ${ }^{\mathrm{TM}}$ polymerase (Applied Biosystems, Foster City, CA, USA) and $4 \mu$ l diluted restriction-ligation mixture by using an initial step of $10 \mathrm{~min}$ at $94{ }^{\circ} \mathrm{C}, 20$ cycles of $20 \mathrm{~s}$ at $94{ }^{\circ} \mathrm{C}, 30 \mathrm{~s}$ at $56^{\circ} \mathrm{C}$, and $2 \mathrm{~min}$ at $72{ }^{\circ} \mathrm{C}$. The amplicons were diluted $1: 20$ in distilled $\mathrm{H}_{2} \mathrm{O}$ and $3 \mu \mathrm{l}$ were subjected to selective PCR using a mastermix of the same concentration as that used for preselective PCR, and adapter-specific primers HindIII + A and
$H h a \mathrm{I}+\mathrm{A}$, containing an additional A nucleotide at the $3^{\prime}$ end. An initial denaturation for 10 min at $94{ }^{\circ} \mathrm{C}$ was followed by a touchdown PCR protocol consisting of (a) 11 cycles of $20 \mathrm{~s}$ each at $94{ }^{\circ} \mathrm{C}, 30 \mathrm{~s}$ annealing starting at $66^{\circ} \mathrm{C}$ and decreasing $1{ }^{\circ} \mathrm{C}$ every cycle until $56{ }^{\circ} \mathrm{C}$ was achieved, and 2 min each at $72{ }^{\circ} \mathrm{C}$ and (b) 19 cycles of $20 \mathrm{~s}$ each at $94{ }^{\circ} \mathrm{C}, 30 \mathrm{~s}$ each at $56^{\circ} \mathrm{C}$, and 2 min each at $72{ }^{\circ} \mathrm{C}$. A final elongation step was performed for $30 \mathrm{~min}$ at $60^{\circ} \mathrm{C}$, after which the samples were cooled to $4{ }^{\circ} \mathrm{C}$. Preselective and selective PCR was performed in a GeneAmp ${ }^{\circledR}$ PCR System 9700 (Applied Biosystems) with the ramp rate set at $90 \%$.

\section{Capillary electrophoresis}

Amplified DNA fragments were separated by capillary electrophoresis on an ABI-310 Genetic Analyzer (Applied Biosystems) with POP4 polymer and GeneScan TAMRA-500 as the internal standard for each sample (Applied Biosystems). After capillary electrophoresis, the ABI Genescan software (Applied Biosystems) was applied. The detection threshold was set at 200 fluorescence units. The AFLP patterns consisted of fragments ranging in size from 50 to 400 bases and were manually edited using the ABI Genotyper software (Applied Biosystems). The TREECON software package (version 1.2, Yves van de Peer, Royal University of Antwerp, Belgium) was applied for distance estimation according to the coefficient of Nei and $\mathrm{Li}$ [28] and cluster analysis using the unweightedpair group method (UPGMA). In accordance with Duim et al. [20], AFLP patterns with at least $90 \%$ similarity were considered to be closely related and were designated as an AFLP type. 
Table 2. PFGE, AFLP, and combined PFGE/AFLP types of C. jejuni isolates from poultry flocks and clinical isolates

\begin{tabular}{|c|c|c|c|c|c|c|c|c|c|c|c|}
\hline $\begin{array}{l}\text { Combined } \\
\text { PFGE/ } \\
\text { AFLP } \\
\text { type }\end{array}$ & Flock & $\begin{array}{l}\text { Proportion } \\
\text { of isolates } \\
\text { per flock } \\
(\%)\end{array}$ & $\begin{array}{l}\text { Number } \\
\text { of human } \\
\text { isolates }\end{array}$ & $\begin{array}{l}\text { PFGE } \\
\text { type }\end{array}$ & $\begin{array}{l}\text { AFLP } \\
\text { type }\end{array}$ & $\begin{array}{l}\text { Combined } \\
\text { PFGE/ } \\
\text { AFLP } \\
\text { type }\end{array}$ & Flock & $\begin{array}{l}\text { Proportion } \\
\text { of isolates } \\
\text { per flock } \\
(\%)\end{array}$ & $\begin{array}{l}\text { Number } \\
\text { of human } \\
\text { isolates }\end{array}$ & $\begin{array}{l}\text { PFGE } \\
\text { type }\end{array}$ & $\begin{array}{l}\text { AFLP } \\
\text { type }\end{array}$ \\
\hline 1 & 1 & 88 & 1 & 1 & 1 & 60 & 24 & 33 & - & 17 & 53 \\
\hline 2 & 1 & 12 & - & 2 & 1 & 61 & 24 & 33 & - & 17 & 54 \\
\hline 3 & 2 & 72 & 9 & 3 & 2 & 62 & 24 & 33 & - & 17 & 55 \\
\hline 4 & 2 & 14 & - & 3 & 3 & 63 & - & - & 11 & 3 & 56 \\
\hline 5 & 2 & 14 & - & 3 & 4 & $64^{*}$ & - & - & 7 & 37 & 39 \\
\hline 6 & 3 & 100 & - & 4 & 5 & 65 & - & - & 5 & 38 & 57 \\
\hline 7 & 4 & 100 & - & 5 & 6 & 66 & - & - & 4 & 39 & 58 \\
\hline 8 & 5 & 33 & - & 6 & 7 & 67 & - & - & 3 & 4 & 59 \\
\hline 9 & 5 & 33 & - & 7 & 8 & 68 & - & - & 3 & 40 & 60 \\
\hline 10 & 5 & 33 & - & 8 & 9 & 69 & - & - & 2 & 41 & 2 \\
\hline 11 & 6 & 10 & - & 9 & 10 & 70 & - & - & 2 & 42 & 61 \\
\hline 12 & 6 & 30 & - & 10 & 11 & 71 & - & - & 2 & 43 & 59 \\
\hline 13 & 6 & 10 & - & 10 & 12 & $72 \dagger$ & - & - & 2 & 44 & 62 \\
\hline 14 & 6 & 10 & - & 9 & 13 & $73 \dagger$ & - & - & 2 & 45 & 63 \\
\hline 15 & 6 & 10 & - & 10 & 14 & $74 \dagger$ & - & - & 2 & 46 & 57 \\
\hline 16 & 6 & 10 & - & 9 & 15 & 75 & - & - & 1 & 3 & 64 \\
\hline 17 & 6 & 20 & - & 10 & 16 & 76 & - & - & 1 & 17 & 65 \\
\hline 18 & 7 & 29 & - & 11 & 17 & 77 & - & - & 1 & 38 & 66 \\
\hline 19 & 7 & 14 & - & 12 & 17 & 78 & - & - & 1 & 44 & 61 \\
\hline 20 & 7 & 14 & - & 12 & 18 & 79 & - & - & 1 & 47 & 67 \\
\hline 21 & 7 & 29 & - & 12 & 19 & 80 & - & - & 1 & 48 & 68 \\
\hline 22 & 7 & 14 & - & 11 & 19 & 81 & - & - & 1 & 49 & 69 \\
\hline 23 & 8 & 30 & - & 8 & 20 & 82 & - & - & 1 & 50 & 62 \\
\hline 24 & 8 & 10 & - & 8 & 21 & 83 & - & - & 1 & 51 & 70 \\
\hline 25 & 8 & 30 & - & 8 & 22 & 84 & - & - & 1 & 52 & 62 \\
\hline 26 & 8 & 10 & - & 13 & 22 & 85 & - & - & 1 & 53 & 62 \\
\hline 27 & 8 & 10 & - & 14 & 23 & 86 & - & - & 1 & 54 & 72 \\
\hline 28 & 8 & 10 & - & 8 & 24 & 87 & - & - & 1 & 55 & 72 \\
\hline 29 & 9 & 13 & - & 15 & 25 & 88 & - & - & 1 & 56 & 73 \\
\hline 30 & 9 & 13 & - & 16 & 26 & 89 & - & - & 1 & 57 & 41 \\
\hline 31 & 9 & 63 & 1 & 17 & 8 & 90 & - & - & 1 & 58 & 74 \\
\hline 32 & 9 & 13 & - & 18 & 27 & 91 & - & - & 1 & 59 & 75 \\
\hline 33 & 10 & 100 & - & 19 & 28 & 92 & - & - & 1 & 60 & 62 \\
\hline 34 & 11 & 100 & - & 20 & 29 & 93 & - & - & 1 & 61 & 76 \\
\hline 35 & 12 & 100 & - & 21 & 30 & 94 & - & - & 1 & 62 & 62 \\
\hline 36 & 13 & 60 & - & 22 & 31 & 95 & - & - & 1 & 63 & 62 \\
\hline 37 & 13 & 20 & - & 23 & 32 & 96 & - & - & 1 & 64 & 77 \\
\hline 38 & 13 & 10 & - & 22 & 33 & 97 & - & - & 1 & 65 & 71 \\
\hline 39 & 13 & 10 & - & 24 & 34 & 98 & - & - & 1 & 66 & 78 \\
\hline 40 & 14 & 100 & - & 25 & 35 & 99 & - & - & 1 & 67 & 41 \\
\hline 3 & 15 & 14 & - & 3 & 2 & 100 & - & - & 1 & 68 & 39 \\
\hline 41 & 15 & 57 & - & 26 & 36 & 101 & - & - & 1 & 69 & 57 \\
\hline 42 & 15 & 29 & - & 3 & 37 & 102 & - & - & 1 & 70 & 79 \\
\hline 43 & 16 & 78 & - & 9 & 38 & 103 & - & - & 1 & 71 & 80 \\
\hline 44 & 16 & 11 & - & 27 & 39 & 104 & - & - & 1 & 72 & 39 \\
\hline 45 & 16 & 11 & - & 28 & 40 & 105 & - & - & 1 & 73 & 81 \\
\hline 46 & 17 & 83 & - & 29 & 41 & 106 & - & - & 1 & 74 & 82 \\
\hline 47 & 17 & 17 & - & 30 & 41 & 107 & - & - & 1 & 75 & 83 \\
\hline 1 & 18 & 83 & - & 1 & 1 & 108 & - & - & 1 & 76 & 59 \\
\hline 48 & 18 & 17 & - & 31 & 42 & 109 & - & - & 1 & 77 & 84 \\
\hline 49 & 19 & 88 & - & 32 & 41 & 110 & - & - & 1 & 78 & 85 \\
\hline 50 & 19 & 13 & - & 33 & 43 & 111 & - & - & 1 & 79 & 62 \\
\hline
\end{tabular}


Table 2. (cont.)

\begin{tabular}{|c|c|c|c|c|c|c|c|c|c|c|c|}
\hline $\begin{array}{l}\text { Combined } \\
\text { PFGE/ } \\
\text { AFLP } \\
\text { type }\end{array}$ & Flock & $\begin{array}{l}\text { Proportion } \\
\text { of isolates } \\
\text { per flock } \\
(\%)\end{array}$ & $\begin{array}{l}\text { Number } \\
\text { of human } \\
\text { isolates }\end{array}$ & $\begin{array}{l}\text { PFGE } \\
\text { type }\end{array}$ & $\begin{array}{l}\text { AFLP } \\
\text { type }\end{array}$ & $\begin{array}{l}\text { Combined } \\
\text { PFGE/ } \\
\text { AFLP } \\
\text { type }\end{array}$ & Flock & $\begin{array}{l}\text { Proportion } \\
\text { of isolates } \\
\text { per flock } \\
(\%)\end{array}$ & $\begin{array}{l}\text { Number } \\
\text { of human } \\
\text { isolates }\end{array}$ & $\begin{array}{l}\text { PFGE } \\
\text { type }\end{array}$ & $\begin{array}{l}\text { AFLP } \\
\text { type }\end{array}$ \\
\hline 51 & 20 & 100 & - & 34 & 44 & 112 & - & - & 1 & 80 & 41 \\
\hline 52 & 21 & 91 & - & 35 & 45 & 113 & - & - & 1 & 81 & 2 \\
\hline 53 & 21 & 9 & - & 35 & 46 & 114 & - & - & 1 & 82 & 62 \\
\hline 54 & 22 & 50 & - & 36 & 47 & 115 & - & - & 1 & 83 & 86 \\
\hline 55 & 22 & 13 & - & 17 & 48 & 116 & - & - & 1 & 84 & 87 \\
\hline 56 & 22 & 13 & - & 36 & 49 & 117 & - & - & 1 & 85 & 41 \\
\hline 57 & 22 & 13 & - & 17 & 50 & 118 & - & - & 1 & 86 & 62 \\
\hline 58 & 22 & 13 & - & 17 & 51 & 119 & - & - & 1 & 87 & 88 \\
\hline 59 & 23 & 100 & - & 10 & 52 & & & & & & \\
\hline
\end{tabular}

* Including a set of three epidemiologically related strains.

$\dagger$ Including a set of two epidemiologically related strains each.

\section{RESULTS}

\section{Prevalence of Campylobacter spp. and species identification in poultry flocks at slaughter}

Thirty of 66 poultry flocks ( $45 \%$ ) tested positive for Campylobacter spp. at slaughter. Positive hydrolysis of hippurate in almost all isolates identified them as C. jejuni. All isolates of 3 flocks as well as 4 isolates of 1 flock failed to hydrolyse hippurate. However, PCR detection of the hip gene identified these isolates as C. jejuni as well.

In 21 flocks, C. jejuni was isolated from all samples; in 3 flocks from $90 \%$, in 1 flock from $80 \%$ and in 2 flocks from $50 \%$ of the samples. Totals of 40,30 , and $10 \%$ of the samples tested positive for C. jejuni in 1 flock each.

\section{Subtyping of $C$. jejuni isolated from poultry}

A total of 177 isolates from 24 flocks were subtyped by both PFGE and AFLP (Table 2).

\section{PFGE}

When combining SmaI and SalI profiles, 36 distinct PFGE types were distinguished. Isolates from 11 flocks (46\%) had only one PFGE type. In $8(33 \%), 4$ (17\%), and 1 (4\%) flock(s) 2, 3, and 4 PFGE types, respectively, were observed. Five types were shared by 2 different flocks each, and 1 type by 3 flocks.

\section{AFLP}

Fifty-five different AFLP types were observed. Ten flocks $(42 \%)$ had only one AFLP type. Multiple
AFLP types were distributed as follows: 3 flocks $(13 \%)$ had 2, 6 flocks ( $25 \%)$ had 3, 2 flocks ( $8 \%$ ) each had 4 and 5, and 1 flock (4\%) had 7 AFLP types. Four genotypes were shared by 2 flocks each.

\section{Combination of PFGE and AFLP}

When combining PFGE and AFLP types, a total of 62 different types were identified. Eight flocks (33\%) contained isolates of only one combined PFGE/ AFLP type. Multiple PFGE/AFLP types were distributed as follows: 5 flocks ( $21 \%$ ) each had 2 and 3, 2 flocks ( $8 \%$ ) each had 4 and 5, and 1 flock (4\%) each had 6 and 7 PFGE/AFLP types. Two PFGE/AFLP types were shared by 2 flocks each.

In some cases, identical AFLP types were further differentiated by PFGE. However, when the criteria for genetic relatedness according to Tenover et al. [29] were applied, they never showed entirely unrelated PFGE profiles. On the other hand, identical PFGE types could more often be further differentiated by AFLP than vice versa, and in some cases did not even cluster together.

\section{Ciprofloxacin resistance of $C$. jejuni isolated from poultry}

One hundred and thirty-nine of $274(50 \%)$ C. jejuni isolates of 30 flocks were resistant to ciprofloxacin. All C. jejuni isolates of 13 flocks ( $43 \%$ ) were sensitive to ciprofloxacin and 13 flocks (43\%) contained resistant isolates only. Four flocks $(14 \%)$ had sensitive as well as resistant isolates, which were clearly identified as different PFGE/AFLP types. 


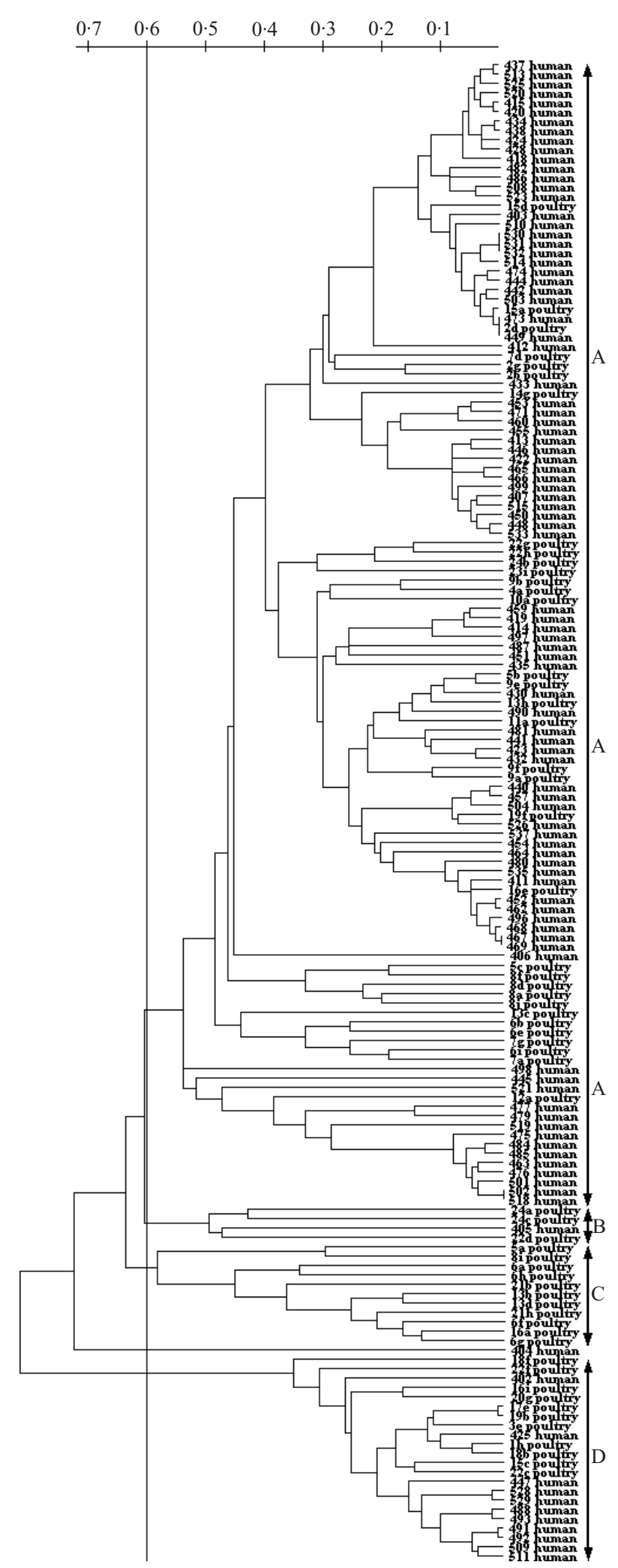

Fig. 1. UPGMA dendrogram of AFLP fingerprints from all human $C$. jejuni isolates included in the study and one poultry isolate per different AFLP type each. At $60 \%$ dissimilarity the isolates are separated in 4 distinct clusters (A-D).

\section{Subtyping of human $C$. jejuni isolates}

One hundred and one clinical $C$. jejuni isolates were subtyped by PFGE and AFLP (Table 2).

PFGE

A total of 55 combined $S m a I / S a l$ I types were identified. Thirteen PFGE types were found in more than one isolate. Type 3 was the predominant type, being observed in 21 isolates $(21 \%)$.

\section{AFLP}

Thirty-eight different AFLP types were found. Thirteen AFLP types were present in more than one isolate. The predominant AFLP type 2 was observed in $12(12 \%)$, and types 56 and 62 in $11(11 \%)$ isolates, respectively.

\section{Combination of PFGE and AFLP}

When combining PFGE and AFLP subtyping data, a total of 60 different PFGE/AFLP types were identified. Thirteen types were shared by different isolates. The predominant PFGE/AFLP types 63 and 3 occurred in $11(11 \%)$ and $9(9 \%)$ isolates, respectively.

Three sets of two and one set of three epidemiologically related isolates were included in the study and could not be distinguished by either subtyping method. One suspected epidemiologically related set of two strains revealed different subtypes with the two methods. Identical AFLP types had completely unrelated PFGE types in many cases. Identical PFGE types yielded, in most cases, either identical AFLP types or AFLP types that clustered together. However, these AFLP clusters also contained isolates with entirely unrelated PFGE types.

\section{Ciprofloxacin resistance of human $C$. jejuni isolates}

Ninety-four of 239 (39\%) of clinical C. jejuni isolates were resistant to ciprofloxacin. Of 101 clinical C. jejuni isolates PFGE and AFLP data were available. With both subtyping methods, some types comprised resistant as well as susceptible isolates. Fifty per cent of the isolates of the PFGE/AFLP types 70 and 71, $40 \%$ of the isolates of type 64 and $33 \%$ of the isolates of types 3 and 67 were resistant to ciprofloxacin.

\section{Comparison of subtyping data of $C$. jejuni isolates from poultry and humans}

Cluster analysis of AFLP data revealed four distinct clusters (A-D) with $60 \%$ dissimilarity (Fig. 1). One 
human isolate (404) did not concur with any cluster group. Human and poultry isolates clustered together throughout the dendrogram, with the exception of cluster $\mathrm{C}$, which contained poultry isolates only.

Four PFGE types and five AFLP types were shared by human and poultry isolates. When combining the results of both subtyping methods, three PFGE/ AFLP types observed in 11 human isolates $(11 \%)$ were shared. PFGE/AFLP type 3 comprised nine human isolates ( 6 of them sensitive and 3 resistant to ciprofloxacin) and ciprofloxacin-resistant isolates of poultry flocks 2 and 15. PFGE/AFLP type 1 consisted of one human isolate and isolates of poultry flocks 1 and 18, all of them being resistant to ciprofloxacin. PFGE/AFLP type 31 comprised ciprofloxacin-sensitive isolates and was shared by one human isolate and isolates from poultry flock 9 .

\section{DISCUSSION}

Campylobacter spp. were found in $45 \%$ of poultry flocks at slaughter in Austria. This rate is lower than that registered in The Netherlands $(>82 \%)$ and the United Kingdom (76\%), but higher than that in Sweden and Norway (27 and $18 \%$, respectively) [3-6]. Probably, the performance of a pre-enrichment step could have increased the isolation rate. However, an increase in the prevalence of Campylobacter spp. to $56 \cdot 8 \%$ during the last 2 years, within flocks sampled in the same area, was reported recently [30]. Both, the predominance of the Campylobacter species C. jejuni observed in the present study and the high percentage of positive isolates per contaminated flock are in accordance with previously published data $[3,4,31,32]$.

With respect to the individual chickens sampled, a single colony was picked for further investigation if the morphology of the colonies was uniform and typical for the expected species. In a few cases two different types of suspected Campylobacter spp. colonies were observed and then a colony of each type was picked. Further analysis of these isolates revealed that one chicken could simultaneously harbour a ciprofloxacin-resistant and a ciprofloxacin-sensitive C. jejuni strain. As only one isolate per chicken was further analysed by PFGE/AFLP it is not known, whether the different types of isolates per chicken observed by ciprofloxacin resistance testing belong to a different PFGE/AFLP type as well.

The combined PFGE/AFLP data revealed multiple infections of flocks with different strains of $C$. jejuni as well as different flocks being infected with strains comprising the same PFGE/AFLP type. Similar results have been obtained with RFLP typing of the $f l a \mathrm{~A}$ and $\mathrm{B}$ genes and serotyping [3, 32-35].

Fifty per cent of $C$. jejuni strains isolated from poultry in Austria were found to be resistant to ciprofloxacin. This figure lies within the range reported in other European countries: $3 \cdot 2-45 \cdot 9 \%$ of Campylobacter spp. and $98.7 \%$ of $C$. jejuni isolated from poultry tested resistant to ciprofloxacin in Ireland, Germany, and Spain, respectively [36-38]. In The Netherlands, $29 \%$ of Campylobacter spp. isolates from poultry were resistant to quinolone [39].

Of the clinical $C$. jejuni isolates tested in the presented study, $39 \%$ were resistant to ciprofloxacin, compared with $22.1 \%$ of clinical isolates of Campylobacter spp. in 1993. In 2000 there was a further rise in resistance to $40 \cdot 2 \%$ [40], the increase being much the same in several countries worldwide $[10,11,37$, $38,41-44]$. In many countries, the prevalence of resistance in clinical strains parallels that in poultry strains. In countries such as Finland, where no quinolones were used in poultry production until 1998 [45], the proportion of human C. jejuni isolates resistant to ciprofloxacin was $2 \cdot 8 \%$ in 1997 [13]. On the other hand, in countries like Spain, where enrofloxacin was licensed for use in poultry production in 1986 [45], $75 \%$ of clinical and $98.7 \%$ of poultry $C$. jejuni isolates were ciprofloxacin resistant in 1997 [38]. The government of Austria has permitted the use of enrofloxacin in poultry since 1989. Unfortunately, there were no data available concerning the use of enrofloxacin in the flocks sampled, but it is commonly used in Austria. Therefore the resistance data revealed in the present study are concordant with this general trend.

When comparing PFGE and AFLP data, we encountered major differences in the ability to subtype C. jejuni isolates of human and poultry origin. With AFLP, many poultry isolates of the same as well as of different flocks showing the same PFGE types were further differentiated, whereas several human isolates representing the same AFLP types showed different PFGE types. DeBoer et al. [46] applied AFLP using the restriction enzymes HindIII and HhaI for subtyping poultry isolates. Their comparison of AFLP data with SmaI-derived PFGE data also showed AFLP to be slightly more discriminative; however, the number of subtyped isolates may have been too small to confirm this trend.

In the present study, comparison of subtyping data revealed three combined PFGE/AFLP types that were 
shared by poultry and clinical isolates. A total of 11 human isolates $(11 \%)$ belonged to this category. With regard to ciprofloxacin resistance, type 3 comprised resistant poultry isolates and resistant as well as sensitive clinical isolates. These data suggest either $(a)$ conversion of a ciprofloxacin-resistant $C$. jejuni strain into a susceptible one during passage in the slaughter house or in the human intestine, which seems unlikely, (b) the presence of resistant and susceptible strains belonging to the same PFGE/AFLP type in poultry flocks, which might have been detected when examining more than 10 chickens per flock, or $(c)$ an unknown common source of infection for both poultry and humans. The small number of subtypes shared by clinical and poultry isolates is in accordance with previously published studies, in which a variety of subtyping techniques were used [13, 19, 20, 22, 36, 47].

Cluster analysis of AFLP data in the present study supports previous reports to the effect that no distinct subpopulations of $C$. jejuni isolates associated with humans or poultry exist [36, 48, 49]. Duim et al. [48] performed AFLP analysis of poultry and human C. jejuni isolates using the same restriction enzymes as those used in the present study. Cluster analysis revealed two subgroups with $40 \%$ genetic homology. In our study, four subgroups containing poultry as well as human isolates were observed. One human isolate was clearly distinguished from these groups.

In conclusion, the present study confirms an increase in ciprofloxacin resistance in $C$. jejuni isolates of human and poultry origin, as observed in several countries worldwide. A small number of human isolates shared PFGE/AFLP types with poultry isolates. However, further studies should also focus on the identification of other sources for C. jejuni infection in humans.

\section{ACKNOWLEDGEMENTS}

The authors would like to thank Mathias Müller, Chair of the Department of Biotechnology and Molecular Genetics, Institute of Animal Breeding and Genetics, University of Veterinary Medicine, Vienna, for general support.

\section{REFERENCES}

1. Feierl G. Nationale Referenzzentrale für Campylobacter: Jahresbericht 2000. Mitt Sanitätsverw 2000; 102: 3-7.

2. Skirrow MB, Blaser MJ. Clinical and epidemiological considerations. In: Nachamkin I, Blaser MJ, Tomkins
LS, eds. Campylobacter jejuni: current status and future trends. Washington, DC: ASM Press, 1992: 3-8.

3. Berndtson E, Emanuelson U, Engvall A, DanielssonTham ML. A 1-year epidemiological study of campylobacters in 18 Swedish chicken farms. Prev Vet Med 1996; 26 : 167-85.

4. Humphrey TJ, Henley A, Lanning DG. The colonization of broiler chickens with Campylobacter jejuni: some epidemiological investigations. Epidemiol Infect 1993; 110: 601-7.

5. Jacobs-Reitsma WF, Bolder NM, Mulder RWAW. Cecal carriage of Campylobacter and Salmonella in Dutch broiler flocks at slaughter: a one-year study. Poultry Science 1994; 73: 1260-6.

6. Kapperud G, Skjerve E, Vik L, et al. Epidemiological investigation of risk factors for campylobacter colonization in Norwegian broiler flocks. Epidemiol Infect 1993; 111 : 243-55.

7. Feierl G, Pschaid A, Sixl B, Marth E. Increase of ciprofloxacin resistance in Campylobacter species in Styria, Austria. Zbl Bakt 1994; 281 : 471-4.

8. Feierl G, Berghold C, Fürpass T, Marth E. Further increase in ciprofloxacin-resistant Campylobacter jejuni/ coli in Styria, Austria. Clin Microbiol Infect 1999; 5: 59-60.

9. Hirschl AM, Wolf D, Berger J, Rotter ML. In vitro susceptibility of Campylobacter jejuni and Campylobacter coli isolated in Austria to erythromycin and ciprofloxacin. Zbl Bakt 1990; 272: 443-7.

10. Endtz HPh, Ruijs GJ, van Klingeren B, Jansen WH, van der Reyden T, Mouton RP. Quinolone resistance in Campylobacter isolated from man and poultry following the introduction of fluoroquinolones in veterinary medicine. J Antimicrob Chemother 1991; 27: 199-208.

11. Smith KE, Besser JM, Hedberg CW, et al. Quinoloneresistant Campylobacter jejuni infections in Minnesota, 1992-1998. N Engl J Med 1999; 20 : 1525-32.

12. Hänninen ML, Perko-Mäkelä P, Pitkälä A, Rautelin H. A three-year study of Campylobacter jejuni genotypes in humans with domestically acquired infections and in chicken samples from the Helsinki area. J Clin Microbiol 2000; 38: 1998-2000.

13. Hänninen ML, Pajarre S, Klossner ML, Rautelin $H$. Typing of human Campylobacter jejuni isolates in Finland by pulsed-field gel electrophoresis. J Clin Microbiol 1998; 36: 1787-9.

14. Iriarte P, Owen RJ. PCR-RFLP analysis of the large subunit (23S) ribosomal RNA genes of Campylobacter jejuni. Lett Appl Microbiol 1996; 23 : 163-6.

15. Jackson CJ, Fox AJ, Wareing DRA, Hutchinson DN, Jones DM. The application of genotyping techniques to the epidemiological analysis of Campylobacter jejuni. Epidemiol Infect 1996; 117: 233-44.

16. Lehner A, Schneck C, Feierl G, et al. Epidemiologic application of pulsed-field gel electrophoresis to an outbreak of Campylobacter jejuni in an Austrian youth centre. Epidemiol Infect 2000; 125: 13-6.

17. Owen RJ, Leeton S. Restriction fragment length polymorphism analysis of the flaA gene of Campylobacter 
jejuni for subtyping human, animal and poultry isolates. FEMS Microbiol Lett 1999; 176: 345-50.

18. Suzuki Y, Ishihara M, Funabashi M, Suzuki R, Isomura S, Yokochi T. Pulsed-field gel electrophoretic analysis of Campylobacter jejuni DNA for use in epidemiological studies. J Infect 1993; 27 : 9-42.

19. On SLW, Nielsen EM, Engberg J, Madsen M. Validity of SmaI-defined genotypes of Campylobacter jejuni examined by SalI, KpnI, and BamHI polymorphisms: evidence of identical clones infecting humans, poultry, and cattle. Epidemiol Infect 1998; 120: 231-7.

20. Duim B, Wassenaar TM, Rigter A, Wagenaar J. Highresolution genotyping of Campylobacter strains isolated from poultry and humans with amplified fragment length polymorphism fingerprinting. Appl Environ Microbiol 1999; 65: 2369-75.

21. Lindstedt BA, Heir E, Vardund T, Melby KK, Kapperud G. Comparative fingerprinting analysis of Campylobacter jejuni subsp. jejuni strains by amplifiedfragment length polymorphism genotyping. J Clin Microbiol 2000; 38: 3379-87.

22. On SLW, Harrington CS. Identification of taxonomic and epidemiological relationships among Campylobacter species by numerical analysis of AFLP profiles. FEMS Microbiol Lett 2000; 193: 161-9.

23. Wegmüller B, Lüthy J, Candrian U. Direct polymerase chain reaction detection of Campylobacter jejuni and Campylobacter coli in raw milk and dairy products. Appl Environ Microbiol 1993; 59: 2161-5.

24. Linton D, Lawson AJ, Owen RJ, Stanley J. PCR detection, identification to species level, and fingerprinting of Campylobacter jejuni and Campylobacter coli direct from diarrheic samples. J Clin Microbiol 1997; 35: 2568-72.

25. National Committee for Clinical Laboratory Standards. Performance standards for antimicrobial susceptibility testing. NCCLS document M2-M6. Wayne: National Committee for Clinical Laboratory Standards, 1997.

26. Link W, Dixkens C, Singh M, Schwall M, Melchinger AE. Genetic diversity in European and Mediterranean faba bean germ plasm revealed by RAPD markers. Theor Appl Genet 1995; 90: 27-32.

27. Lan R, Reeves PR. Unique adaptor design for AFLP fingerprinting. BioTech 2000; 29: 745-50.

28. Nei M, Li WH. Mathematical model for studying genetic variations in terms of restriction endonucleases. Proc Natl Acad Sci USA 1979; 76: 5269-73.

29. Tenover FC, Arbeit RD, Goering RV, et al. Interpreting chromosomal DNA restriction patterns produced by pulsed-field gel electrophoresis: criteria for bacterial strain typing. J Clin Microbiol 1995; 33 : 2233-9.

30. Ursinitsch B, Pless P, Köfer J. Prevalence and resistance behaviour of Campylobacter spp. in faecal and product samples of Styrian broiler flocks. 11th International Workshop on Campylobacter, Helicobacter. 2001; abstr. E-25.

31. Evans SJ, Sayers AR. A longitudinal study of Campylobacter infection of broiler flocks in Great Britain. Prev Vet Med 2000; 46: 209-23.
32. Jacobs-Reitsma WF. Aspects of epidemiology of Campylobacter in poultry. Vet Quart 1997; 19: 113-7.

33. Shreeve JE, Toszeghy M, Pattsion M, Newell DG. Sequential spread of Campylobacter infection in a multipen broiler house. Avian Dis 2000; 44: 983-8.

34. Stern NJ, Myszewski MA, Barnhart HM, Dreesen DW. Flagellin A gene restriction fragment length polymorphism patterns of Campylobacter spp. isolates from broiler production sources. Avian Dis 1997; 41: 899-905.

35. Van de Giessen A, Mazurier SI, Jacobs-Reitsma W, et al. Study on the epidemiology and control of Campylobacter jejuni in poultry broiler flocks. Appl Environ Microbiol 1992; 58: 1913-7.

36. Lucey B, Feurer C, Greer P, Moloney P, Cryan B, Fanning S. Antimicrobial resistance profiling and DNA amplification fingerprinting (DAF) of thermophilic Campylobacter spp. in human, poultry and porcine samples from the Cork region of Ireland. J Appl Microbiol 2000; 89: 727-34.

37. Atanassova V, Ring C. Campylobacter spp. im Rahmen der Geflügelfleischgewinnung - Vorkommen und Chinolon-Resistenz. Zbl Hyg Umweltmed 1997/98; 200: 542-52.

38. Sáenz Y, Zarazaga M, Lantero M, Gastañares MJ, Baquero F, Torres C. Antibiotic resistance in Campylobacter strains isolated from animals, foods, and humans in Spain in 1997-1998. Antimicrob Agents Chemother 2000; 44: 267-71.

39. Jacobs-Reitsma WF, Koenraad PMFJ, Bolder NM, Mulder RWAW. In vitro susceptibility of Campylobacter and Salmonella isolates from broilers to quinolones, ampicillin, tetracycline, and erythromycin. Vet Quart 1994; 16: 206-8.

40. Feierl G, Wagner U, Sixl B, Grisold A, Daghofer E, Marth E. Epidemiology of campylobacteriosis and development of resistance in Styria, Austria. 11th International Workshop on Campylobacter, Helicobacter. 2001; abstr. B-15.

41. Gaudreau Ch, Gilbert H. Antimicrobial resistance of clinical strains of Campylobacter jejuni subsp. jejuni isolated from 1985 to 1997 in Quebec, Canada. Antimicrob Agents Chemother 1998; 42: 2106-8.

42. Gaunt PN, Piddock LJV. Ciprofloxacin resistant Campylobacter spp. in humans: an epidemiological and laboratory study. J Antimicrob Chemother 1996; 37: 747-57.

43. Sánchez R, Fernández-Baca V, Díaz MD, Muñoz $\mathrm{P}$, Rodríguez-Créixems M, Bouza E. Evolution of susceptibilities of Campylobacter spp. to quinolones and macrolides. Antimicrob Agents Chemother 1994; 38: 1879-82.

44. Thwaites RT, Frost JA. Drug resistance in Campylobacter jejuni, C. coli, and C. lari isolated from humans in North West England and Wales, 1997. J Clin Pathol 1999; 52: 812-4.

45. Engberg J, Aarestrup FM, Taylor DE, Gerner-Smidt P, Nachamkin I. Quinolone and macrolide resistance in Campylobacter jejuni and C. coli: resistance mechanisms and trends in human isolates. Emerg Infect Dis $2001 ; 7: 24-34$. 
46. DeBoer P, Duim B, Rigter A, van der Plas J, JacobsReitsma WF, Wagenaar JA. Computer-assisted analysis and epidemiological value of genotyping methods for Campylobacter jejuni and Campylobacter coli. J Clin Microbiol 2000; 38: 1940-46.

47. Møller-Nielsen E, Engberg J, Fussing V, Petersen L, Brogren $\mathrm{CH}$, On SLW. Evaluation of phenotypic and genotypic methods for subtyping Campylobacter jejuni isolates from humans, poultry, and cattle. J Clin Microbiol 2000; 38: 3800-10.
48. Duim B, Ang CW, van Belkum A, et al. Amplified fragment length polymorphism analysis of Campylobacter jejuni strains isolated from chickens and from patients with gastroenteritis or Guillain-Barré or Miller Fisher Syndrome. Appl Environ Microbiol 2000; 66: 3917-23.

49. Hilton AC, Mortiboy D, Banks JG, Penn ChW. RAPD analysis of environmental, food and clinical isolates of Campylobacter spp. FEMS Immun Med 1997; Microbiol 18: 119-24. 